\title{
Transcranial doppler findings in a case of rabies encephalitis
}

\author{
Aarti Sarwal ${ }^{1 *}$, Joshua Botdorf ${ }^{2}$, Christopher R. Newey ${ }^{3}$ and Manjamalai Sivaraman ${ }^{4}$ \\ *Correspondence: asarwal@wakehealth.edu

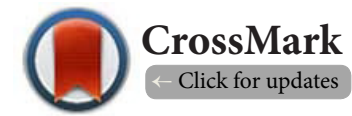 \\ 'Department of Anesthesiology, Section on Critical Care, Wake Forest School of Medicine, Winston-Salem, North Carolina, USA. \\ ${ }^{2}$ Division of Nephrology, Department of Internal Medicine, University of Missouri, Columbia, USA. \\ ${ }^{3}$ Department of Neurology, University of Chicago, $5841 \mathrm{~S}$. Maryland Avenue, Chicago, USA. \\ ${ }^{4}$ Department of Neurology, University of Missouri, Columbia, USA.
}

\begin{abstract}
Rabies encephalitis is a uniformly fatal disease with several hypothesis proposed to explain pathogenesis and mortality. One such theory premises deficiency of neuronal nitric oxide synthetase deficiency causing selective cerebral vasospasm. We present transcranial Doppler findings in a case of rabies that did not show imaging evidence of vasospasm. Transcranial Doppler in two published cases have reported occurrence of global vasospasm. Our case showed no sonographic vasospasm in middle cerebral arteries but consistently increased distal resistance indices likely due to cerebral edema. While vasospasm may occur in rabies, cerebral edema secondary to disease or pharmacological interventions confounds transcranial Doppler readings. Cerebral angiogram and multimodality monitoring is encouraged.
\end{abstract}

Keywords: Rabies encephalitis, vasospasm, transcranial doppler, cerebral edema

\section{Introduction}

Rabies in humans is a fatal illness characterized by severe encephalopathy and generalized paresis. When the disease is not treated, death typically occurs within five to seven days after the onset of symptoms. Rabies virus is not cytopathic and does not incite inflammatory response [1]. Several theories have been hypothesized to explain pathogenesis in rabies that leads to mortality. Recent reports have implicated dysautonomia and cerebral artery vasospasm [2]. With intensive care and experimental protocols, cases of rabies encephalitis are surviving longer, giving us an opportunity to assess pathophysiological changes that occur in rabies. This can help tailor further strategies in our effort to find a treatment against this uniformly fatal illness. We managed a case of rabies encephalitis with institution of the Milwaukee protocol [3]. This is an experimental protocol involving treatment with an intense antiexcitoxic strategy with use of therapeutic coma with GABA agonists and NMDA receptor antagonists. Use of this strategy was reported in survival of one case though widespread application has been limited by lack of reproducibility of similar clinical results. We report the neuroimaging findings in our case that was managed using this protocol and monitored with transcranial Doppler (TCD) ultrasound.

\section{Case presentation}

Our patient was a 55-year-old male who presented to the emergency department with acute onset hydrophobia, ataxia, tremors, and paresthesias five weeks after a bat bite to the ear. His symptoms progressed over five days with deterioration in mental status requiring intubation and sedation. Cerebrospinal fluid (CSF), serum, saliva, and nuchal skin specimens were sent for rabies virology to Center for Disease Control while a clinical diagnosis of rabies encephalitis was entertained. Initial work-up for other etiologies including metabolic profile, CSF analysis, and computed tomography (CT) head scan was unremarkable. After discussions with family about the clinical suspicion of rabies, fatal course of the illness and experimental therapies, one such protocol called the Milwaukee Protocol was initiated on the 5 th day of onset of symptoms. This initial regimen involved sedation with ketamine and benzodiazepines. Amantadine, nimodipine, tetrahydrobiopterin (BH4), coenzyme Q10, multivitamins, levocarnitine were later started to prevent cerebral vasospasm as suggested by the Milwaukee protocol. Continuous electroencephalogram (EEG) monitoring was initiated to titrate benzodiazepines dose to induce therapeutic coma. Serial TCD studies were obtained with baseline values obtained on day of admission, 5 days since onset of symptoms 
Sarwal et al. Journal of Medical Disorders 2014,

http://www.hoajonline.com/journals/pdf/2053-3659-2-2.pdf

doi: $10.7243 / 2053-3659-2-2$

and same day as initiation of ketamine and amantadine (Table 1). Baseline TCD (@ pCO 30.6, mean arterial pressure [MAP] 96) showed normal bilateral middle cerebral artery (MCA) velocity but high pulsatility indices (PI). Autonomic failure ensued and sedatives were weaned as EEG progressed to burst suppression spontaneously. Vasopressors were started for hypotension and $\mathrm{BH} 4$ was started. Magnetic resonance imaging (MRI) was planned but not done due to hemodynamic instability. Follow-up TCD (@pCO 43.4, MAP 71) showed mildly increased but normal mean flow velocities and high resistance indices in bilateral MCA. A CT-angiogram head done on Day 4 of admission showed diffuse effacement of sulci but no focal ischemic stroke or cerebral vasospasm. Lumbar puncture done the same day had opening pressure of $41 \mathrm{~cm} \mathrm{H}_{2} \mathrm{O}$. Follow-up TCD the next day was remarkable for decreased mean flow velocity with marked increase in pulsatility index on the right side. Salt tablets were started for inducing hypernatremia along with diuresis to counteract increasing cerebral edema. A second vasopressor was added to maintain cerebral perfusion pressures. External ventricular drainage was deferred given grim prognosis and high risk of transmission. Over the next day, patient developed acute renal failure, severe acidosis followed by hypotension refractory to vasopressors, and transtentorial herniation despite maximal osmotic therapy. After family discussions based on protracted course, comfort care measures were initiated. Patient died on Day 8 of hospitalization.

\section{Literature review}

We searched Ovid Medline and Pubmed for MeSH terms "rabies encephalitis." Abstracts were manually reviewed to search for neuroimaging, sonographic, and angiographic findings. References listed in journals were also searched for neuroimaging findings.

Neuroimaging features in rabies encephalitis have been reported only in a few case reports [5-9]. Nonenhancing, ill-defined, mild hyperintensity changes in the brain stem, hippocampi, hypothalami, deep and subcortical white matter, and deep and cortical gray matter were demonstrated on T2-weighted images in non-comatose patients with rabies [5]. Enhancement with gadolinium-based contrast material was seen at the hypothalami, brain stem nuclei, spinal cord gray matter, and intradural cervical nerve roots only when the patients became comatose [5].

We found TCD studies in two reported cases that described global vasospasm [2]. Willougby et al., reported two rabies cases with deficiencies of $\mathrm{BH} 4$ and associated neurotransmitters like L-arginine where flow velocities and resistivity indices (RI) or pulsatility indices (PI) of MCA were obtained by TCD. In the first case of a child with bat-associated rabies, they described severe bilateral MCA spasm with maximum velocity of $400 \mathrm{~cm} / \mathrm{s}$ on hospital day (HD)-10 that responded to very low dose $(0.2 \mathrm{mcg} / \mathrm{kg} / \mathrm{min})$ nitroprusside. Patient developed cerebral herniation but survived. $\mathrm{BH} 4$ and L-arginine were started with follow-up TCD showing fall in max MCA velocity, high RI on HD-17 with zero intracranial pressure. Arginine vasopressin and nitroprusside were given. Patient went on to develop increased cerebral edema from cerebral venous sinus thrombosis and died on HD-27. The second published case described a child with diagnosed dog-associated rabies who suffered cardiac arrest. After being resuscitated, his TCDs showed high mean velocity on MCA and internal carotid

Table 1. Transcranial doppler findings*.

\begin{tabular}{|c|c|c|c|c|c|c|c|c|c|c|c|c|c|c|c|c|c|c|c|c|c|}
\hline \multicolumn{4}{|c|}{$\begin{array}{l}\text { Physiological } \\
\text { parameters at the } \\
\text { time of TCD }\end{array}$} & \multicolumn{5}{|c|}{ Left MCA } & \multicolumn{5}{|c|}{ Right MCA } & \multicolumn{3}{|c|}{ Left ICA } & \multicolumn{3}{|c|}{ Right ICA } & \multicolumn{2}{|c|}{ Lindegaard } \\
\hline Day & $\mathrm{pCO}_{2}$ & MAP & BIS & PSV & EDV & Mean & RI & PI & PSV & EDV & Mean & RI & PI & PSV & EDV & Mean & PSV & EDV & Mean & Left & Right \\
\hline $\begin{array}{l}\text { Onset } \\
11 / 19\end{array}$ & -- & -- & -- & -- & -- & -- & -- & -- & -- & -- & -- & -- & -- & -- & -- & -- & -- & -- & -- & -- & -- \\
\hline $\begin{array}{l}\text { Day } 1 \\
11 / 24\end{array}$ & 30.6 & 96 & -- & 108 & 28 & 47 & 0.74 & 1.70 & 83 & 23 & 37 & 0.72 & 1.62 & 70 & 18 & 38 & 54 & 17 & 27 & 1.24 & 1.37 \\
\hline $\begin{array}{l}\text { Day } 3 \\
11 / 26\end{array}$ & 57.2 & 68 & -- & 121 & 35.2 & 64 & 0.71 & 1.34 & 113 & 34.4 & 61 & 0.70 & 1.29 & -- & -- & -- & -- & -- & -- & -- & -- \\
\hline $\begin{array}{l}\text { Day } 4 \\
11 / 27\end{array}$ & 43.4 & 71 & 60 & 181 & 39 & 70 & 0.78 & 2.03 & 161 & 39.5 & 65 & 0.75 & 1.87 & -- & -- & -- & -- & -- & -- & -- & -- \\
\hline $\begin{array}{l}\text { Day } 5 \\
11 / 28\end{array}$ & 54.3 & 69 & 65 & 141 & 37.6 & 70.1 & 0.73 & 1.48 & 114 & 32 & 55.3 & 0.72 & 1.48 & -- & -- & -- & -- & -- & -- & -- & -- \\
\hline $\begin{array}{l}\text { CTA } \\
11 / 28\end{array}$ & -- & -- & -- & -- & -- & -- & -- & -- & -- & -- & -- & -- & -- & -- & -- & -- & -- & -- & -- & -- & -- \\
\hline $\begin{array}{l}\text { Day } 6 \\
11 / 29\end{array}$ & 55.9 & 68 & 14 & 135 & 25 & 56 & 0.81 & 1.96 & 82 & 0 & 19 & 1 & 4.32 & 165 & 44 & -- & 167 & 85 & -- & 0.82 & 0.49 \\
\hline
\end{tabular}

${ }^{\star}$ Day 1 represents first day of transcranial Doppler (TCD) values available. MCA: Middle cerebral artery; ICA: Internal carotid artery; pCO; Partial pressure $\mathrm{CO}_{2}$ on arterial blood gas; MAP: Mean arterial pressure; BIS: Bispectral index values; PSV: Peak systolic velocity; EDV: End diastolic velocity; RI: Resistivity index; PI: Pulsatility index. Lindegaard ratio or interhemispheric index is the ratio between mean flow velocities in the middle cerebral artery and the distal extracranial internal carotid artery. 
artery (ICA) that normalized on HD-6 after initiation of $\mathrm{BH} 4$. Follow TCDs on HD-11 showed decreased mean flow velocities but high RI with no cerebral edema on CT head or lumbar puncture. Hospital course was complicated with seizures, cerebral edema, and death.

Angiographic findings have been reported in two cases of rabies encephalitis of which only one showed vasospasm $[7,10]$. Sing et al., described a 10-year-old girl with clinical features of an acute encephalitic process. Cerebral angiogram showed narrowing at the supraclinoid ICA and terminal basilar arteries [7]. A diagnosis of rabies was established at autopsy. Although the major blood vessels and basal meninges were normal it was possible that transient arterial spasm induced by the viral infection was responsible for the angiographic appearance. Another case report discussed rabies encephalitis through a bat bite but angiogram was reported normal [10].

\section{Discussion}

Rabies is known to be uniformly fatal for humans. Some experimental therapies have been proposed to delay death once a patient becomes symptomatic. One such protocol, popularly called the "Milwaukee Protocol," was considered for our patient. This protocol was first used in 1995 resulting in recovery of a patient who is still alive and functioning [3]. It has subsequently been attempted in multiple other patients worldwide with various modifications. None of these patients survived. The rabies virus is not cytopathic and does not incite inflammatory response [1]. Several theories have been hypothesized to explain pathogenesis in rabies that leads to mortality. One such theory hypothesizes that rabies virus causes selective deficiency of $\mathrm{BH} 4$ leading to $\mathrm{nNOS}$ deficiency causing selective cerebral vasospasm. Rabies virus, which almost exclusively targets neurons, disproportionately affects cerebral over systemic perfusion by disrupting nNOS that causes cerebral artery spasm. It is believed that the morbidity and mortality burden of rabies stems from cerebral vasospasm or from dysautonomia through neuropathy $[2,4]$. The combination of drugs and treatment strategies in Milwaukee Protocol are aimed to reverse this process to allow latent immune mechanisms to clear rabies virus from the body. Though the success of this protocol or any other therapy has not been reproduced, the prolonged survival of rabies cases is bringing to light several interesting findings.

Transcranial Doppler provides indirect information on cerebral hemodynamics. The diagnostic reliability of TCD for detecting vasospasm using single velocities is low, and thus could generate false-positive results attributable to hyperemia and/or hyperperfusion. Several authors have reported high specificity (94-100\%) and low sensitivity (39-91\%) for vasospasm detection in the MCA when applying the complete definition of sonographic vasospasm. Several conditions often associated with an increase in cerebral blood flow (e.g., seizures, fever, and mental activity) can increase arterial velocity at the base of the skull without reducing vessel diameter.
False negative results may be seen in distal vasospasm or systemic hypoperfusion. Some authors have suggested use of PI, RI, or Lindegaard ratios to increase the sensitivity and help delineate vasospasm. In our patient, mean flow velocities remained within normal limits though Pls were remarkably high. High pulsatility and Rls correlate well in this case $(r=0.99)$ and may represent peripheral vascular resistance secondary to distal vasospasm or progressive cerebral edema. Increased intracranial pressures in this case may have been a side effect of vasodilator therapy, hypervolemia or be part of natural history of rabies adding to spectrum of Doppler findings in this rare disease. We were unable to perform further testing like cerebral angiogram due to patient instability.

\section{Conclusion}

This case highlights a patient with rabies encephalitis managed with the "Milwaukee Protocol" who was monitored with TCD and CTA. A review of literature only provided two other cases of published reports of TCD findings and only one with cerebral angiographic findings in this uniformly fatal disease. Considering the proposed hypothesis implicating cranial artery vasospasm as a cause of mortality in rabies, understanding the spectrum of patterns of TCD findings can prove to be very valuable. The critical multisystem organ involvement, complex nature of therapeutic interventions done in this case and limitations of available testing confound definitive conclusions. Use of TCD in early monitoring of any diagnosed cases of rabies encephalitis is encouraged to help further the understanding of this disease.

\section{Competing interests}

The authors declare that they have no competing interests.

Authors' contributions

\begin{tabular}{|l|c|c|c|l|}
\hline Authors' contributions & AS & JB & CN & MS \\
\hline Research concept and design & $\checkmark$ & -- & -- & -- \\
\hline Collection and/or assembly of data & $\checkmark$ & $\checkmark$ & -- & -- \\
\hline Data analysis and interpretation & $\checkmark$ & -- & -- & -- \\
\hline Writing the article & $\checkmark$ & -- & $\checkmark$ & -- \\
\hline Critical revision of the article & $\checkmark$ & $\checkmark$ & -- & $\checkmark$ \\
\hline Final approval of article & $\checkmark$ & $\checkmark$ & $\checkmark$ & -- \\
\hline Statistical analysis & $\checkmark$ & -- & -- & -- \\
\hline
\end{tabular}

\section{Acknowledgement}

The authors would like to thank nursing and paramedical staff of the University Hospital at University of Missouri, Columbia, USA and all of those who helped to take care of this patient.

\section{Publication history}

Editor: Ambuja S Bale, National Center for Environmental Assessment, USA.

EIC: Gianluca Serafini, Sapienza University of Rome, Italy. Received: 26-Aug-2014 Final Revised: 14-Sep-2014

Accepted: 23-Sep-2014 Published: 30-Sep-2014

\section{References}

1. Johnson $N$, Cunningham AF and Fooks AR. The immune response to rabies virus infection and vaccination. Vaccine. 2010; 28:3896-901. | 
Sarwal et al. Journal of Medical Disorders 2014,

http://www.hoajonline.com/journals/pdf/2053-3659-2-2.pdf

Article I PubMed

2. Willoughby RE, Roy-Burman A, Martin KW, Christensen JC, Westenkirschner DF, Fleck JD, Glaser C, Hyland K and Rupprecht CE. Generalised cranial artery spasm in human rabies. Dev Biol (Basel). 2008; 131:367-75. | PubMed

3. Willoughby RE, Jr., Tieves KS, Hoffman GM, Ghanayem NS, Amlie-Lefond $\mathrm{CM}$, Schwabe MJ, Chusid MJ and Rupprecht CE. Survival after treatment of rabies with induction of coma. N Engl J Med. 2005; 352:2508-14. | Article I PubMed

4. Mitrabhakdi E, Shuangshoti S, Wannakrairot P, Lewis RA, Susuki K, Laothamatas J and Hemachudha T. Difference in neuropathogenetic mechanisms in human furious and paralytic rabies. J Neurol Sci. 2005; 238:3-10. | Article | PubMed

5. Laothamatas J, Hemachudha T, Mitrabhakdi E, Wannakrairot P and Tulayadaechanont $\mathrm{S}$. MR imaging in human rabies. AJNR Am J Neuroradiol. 2003; 24:1102-9. | Article | PubMed

6. Awasthi $\mathrm{M}$, Parmar $\mathrm{H}$, Patankar T and Castillo $\mathrm{M}$. Imaging findings in rabies encephalitis. AJNR Am J Neuroradiol. 2001; 22:677-80. | Article | PubMed

7. Sing TM and Soo MY. Imaging findings in rabies. Australas Radiol. 1996; 40:338-41. | Article | PubMed

8. Rao AS, Varma DR, Chalapathi Rao MV and Mohandas S. Case Report: Magnetic resonance imaging in rabies encephalitis. Indian J Radiol Imaging. 2009; 19:301-4. | Article | PubMed Abstract | PubMed Full Text

9. Pleasure $\mathrm{SJ}$ and Fischbein NJ. Correlation of clinical and neuroimaging findings in a case of rabies encephalitis. Arch Neurol. 2000; 57:1765-9. | Article | PubMed

10. Roine RO, Hillbom M, Valle M, Haltia M, Ketonen L, Neuvonen E, Lumio J and Lahdevirta J. Fatal encephalitis caused by a bat-borne rabies-related virus. Clinical findings. Brain. 1988; 111:1505-16. | Article | PubMed

\section{Citation:}

Sarwal A, Botdorf J, Newey CR and Sivaraman M. Transcranial doppler findings in a case of rabies encephalitis. J Med Disord. 2014; 2:2.

http://dx.doi.org/10.7243/2053-3659-2-2 